\title{
Erratum
}

Polymer Bulletin 6, 541-546 (1982)

\section{Revisal of the Chain Equilibration Process in Strained Polymer Melts}

\section{Pierre Thirion}

Laboratoire P.C.S.M., 10 rue Vauquelin, F-75231 Paris Cedex 05, France

In equation [1], expressing the stress tensor of the "equivalent quasi equilibrium network", the summation $\Sigma$ of the tensorial term from $i=1$ to $N$ should be replaced by the average of this term over all orientations of the unit vector $U$ in the equilibrium state.

Moreover, $N(t)$ means here the number of chain segments and $n_{j}(t)$ the number of mononers within each segment $i$, which both remain at time $t$.

Responsible for the text: The Editors (see inside title page of this issue). For advertisements: E. Iückermann, Kurfürstendamm 237, D-1000 Berlin 15, Tel. (030) 882 1031, Telex 01-85411, Springer-Verlag Berlin Heidelberg New York Printed in Germany by Beltz Offsetdruck, Hemsbach/Bergstraße (C) Springer-Verlag GnbH \& Co KG Berlin Heidelberg 1982 